Pacific Journal of Mathematics

ON UNCONDITIONALLY CONVERGING SERIES AND 


\title{
ON UNCONDITIONALLY CONVERGING SERIES AND BIORTHOGONAL SYSTEMS IN A BANACH SPACE
}

\author{
Gregory F. Bachelis and Haskell P. Rosenthal
}

Our main result is as follows: Let $B$ be a Banach space containing no subspace isomorphic (linearly homeomorphic) to $l_{\infty}$, and let $\left\{\left(b_{n}, \beta_{n}\right)\right\}$ be a biorthogonal sequence in $B$ such that $\left(\beta_{n}\right)$ is total. If $x \in B$ then $\sum_{n=1}^{\infty} \beta_{n}(x) b_{n}$ converges unconditionally to $x$ if and only if for every sequence $\left(a_{n}\right)$ of 0 's and l's there exists $y \in B$ with $\beta_{n}(y)=a_{n} \beta_{n}(x)$ for all $n$. This theorem improves previous results of Kadec and Pelczynski.

Similar results are obtained in the context of biorthogonal decompositions of a Banach space into separable subspaces.

1. Preliminaries. We follow the notation of [2] for the most part, and we also refer the reader to [2] for various results concerning unconditional convergence. We recall that a sequence of pairs $\left\{\left(b_{n}, \beta_{n}\right)\right\}$ is called a biorthogonal sequence in the Banach space $B$ if for all $m$ and $n, b_{m} \in B, \beta_{n} \in B^{*}$, and $\beta_{m}\left(b_{n}\right)=\delta_{m n} ;\left(\beta_{n}\right)$ is said to be total (in $B$ ) if given $x \in B$ with $\beta_{n}(x)=0$ for all $n$, then $x=0$. Finally, we denote the space of all bounded scalar-valued sequences by $l_{\infty}$.

2. The Main Result. We first need the following lemma, due to Seever [8]:

Lemma 1. Let $X$ be a Banach space and $T: X \rightarrow l_{\infty}$ be a bounded linear map such that for every $a \in l_{\infty}$ with $a_{n}=0$ or 1 for all $n$, there exists $x \in X$ with $T x=a$. Then $T(X)=l_{\infty}$.

Proof. Our hypotheses imply that $T$ has dense range; thus it is enough to show that $T$ has closed range. If not, then $T^{*}$ does not have closed range, so there exists a sequence $\left(\gamma_{n}\right)$ in $l_{\infty}^{*}$ with $\left\|\gamma_{n}\right\| \rightarrow \infty$ and $\left\|T^{*}\left(\gamma_{n}\right)\right\|=1$ for all $n$. But if $a \in l_{\infty}$ and $a_{n}=0$ or 1 for all $n$, then choosing $x \in X$ with $T x=a$, we have that

$$
\sup _{n}\left|\gamma_{n}(a)\right|=\sup _{n}\left|T^{*} \gamma_{n}(x)\right| \leqq\|x\|<\infty \text {. }
$$

Thus identifying $l_{\infty}$ with $C(\beta N)$ (the space of continuous scalar-valued functions on the Stone-Cèch compactification of $N$ ) and each $\gamma_{n}$ with a complex regular Borel measure on $\beta N$, we have by a theorem of Dieudonne [3] (c.f. also the Correstion, pp. 311-313 of [7]) that 
$\sup _{n}\left\|\gamma_{n}\right\|<\infty$, a contradiction.

THEOREM 1. Let $B$ be a Banash spase containing no subspase isomorphic to $l_{\infty}$, and let $\left\{\left(b_{n}, \beta_{n}\right)\right\}$ be a biorthogonal sequence in $B$ such that $\left(\beta_{n}\right)$ is total. Let $x \in B$. Then

(1) $\sum_{n=1}^{\infty} \beta_{n}(x) b_{n}$ converges unsonditionally to $x$

$$
\text { if and only if }
$$

(2) Given $a \in l_{\infty}$ with $a_{n}=0$ or 1 for all $n$, there exists $y \in B$ such that $\beta_{n}(y)=a_{n} \beta_{n}(x)$ for all $n$.

Proof. Let $x \in B$. If $\sum \beta_{n}(x) b_{n}$ converges unconditionally, then it is subseries convergent; thus " $(1) \Rightarrow(2)$ " is immediate. Now suppose that (2) holds. We shall prove that $\Sigma \beta_{n}(x) b_{n}$ converges unconditionally. Since $\left(\beta_{n}\right)$ is total in $B$ it then follows that the limit is $x$.

Let $M$ be the set of all $a \in l_{\infty}$ such that there exists $y \in B$ with $\beta_{n}(y)=a_{n} \beta_{n}(x)$ for all $n$. Given such an $a$, there is a unique $y$ satisfying the above. We then define $\|a\|=\|a\|_{\infty}+\|y\|$. It is easily verified that $M$ is a Banach space under this norm. Thus the inclusion map $T: M \rightarrow l_{\infty}$ is continuous and satisfies the hypotheses of Lemma 1 . Hence $M=l_{\infty}$, so $T^{-1}$ is continuous. Thus the mapping $U$ given by $\beta_{n}(U(\alpha))=a_{n} \beta_{n}(x)$ for all $n$, is a continuous linear mapping of $l_{\infty}$ into the Banach space $B$, which by hypothesis contains no subspace isomorphic to $l_{\infty}$. Hence by [7, Cor. 1.4], $U$ is weakly compact.

Given a subseries $\sum_{k} \beta_{n_{k}}(x) b_{n_{k}}$, let $a$ be the characteristic function of $\left(n_{k}\right)$. If a subsequence of the partial sums of this subseries, $\left(S_{k}\right)$, converges weakly to $z \in B$, then $\beta_{n}(z)=\lim _{k-\infty} \beta_{n}\left(S_{k}\right)=a_{n} \beta_{n}(x)$ for all $n$; thus $U(a)=z$. Since the partial sums of this subseries are contained in a weakly sequentially compact set (the image under $U$ of the unit ball of $l_{\infty}$ ), it follows that the subseries itself converges weakly to $U(a)$. Hence $\Sigma \beta_{n}(x) b_{n}$ is weakly subseries convergent, so by the Orlicz-Pettis Theorem it is unconditionally convergent.

REmarks. (I) If $B$ is separable, then $B$ contains no subspace isomorphic to the (nonseparable) space $l_{\infty}$, so Theorem 1 holds. In this case one can apply a theorem of Grothendieck [5, p. 168] in the proof, rather than the generalization given by [7, Cor. 1.4].

(II) Suppose that $B$ is separable. Kadec and Pelczynski proved the equivalence of (1) and (2) under the above hypotheses together with the added assumption that the norm $\|x\|=\sup \left\{\left|x^{*}(x)\right|\right\}$ (the supremum taken over $x^{*}$ in the linear span of $\left(\beta_{n}\right)$ with $\left\|x^{*}\right\| \leqq 1$ ), is equivalent to the original norm of $B$. They also proved that $\Sigma \beta_{n}(x) b_{n}$ converges unconditionally to $x$ if for all $a \in l_{\infty}$ there exists $y \in B$ such that $\beta_{n}(y)=a_{n} \beta_{n}(x)$ for all $n$, [6, Thms. 4 and 5, resp.]

(III) An earlier version for Theorem 1 contained the unnecessary 
hypothesis that $\left(b_{n}\right)$ be fundamental in $B$. The authors are indebted to Professor Ivan Singer for pointing this out.

(IV) It is crucial that $B$ contain no subspace isomorphic to $l_{\infty}$, since if $B$ equals $l_{\infty}$ itself, then the obvious biorthogonal system satisfies (2) for all $x \in B$. The assumption that the biorthogonal set of pairs be denumerable, however, is irrelevant; see Remark (I) at the end of the paper. It is also crucial that $\left(\beta_{n}\right)$ be total, for consider the following biorthogonal sequence $\left\{\left(b_{n}, \beta_{n}\right)\right\}$ in a separable Hilbert space $H$ :

Let $\left(e_{n}\right)$ be a complete orthonormal sequence in $H$; let $\left(y_{n}\right)$ be a sequence such that for each $n$ there are infinitely many indices $m$ such that $y_{m}=y_{n}$, such that $y_{2}=y_{2 j}$ for all $j$, and such that $\left\{y_{n}: n=1,2, \cdots\right\}=\left\{e_{2 n-1}: n=1,2, \cdots\right\} ;$ put $b_{n}=e_{2 n}+y_{n}$ and $\beta_{n}=e_{2 n}^{*}$ for all $n$ (where $\left.e_{2 n}^{*}(x)=\left\langle x, e_{2 n}\right\rangle, x \in H\right)$. Now let $x=\sum_{n=1}^{\infty}(1 / n) e_{4 n}$. Then the span of $\left(b_{n}\right)$ is dense in $H$, yet

(i) for every $a \in l_{\infty}$ there exists $y \in H$ with $\beta_{n}(y)=a_{n} \beta_{n}(x)$ for all $n$, and

(ii) $\lim _{n \rightarrow \infty}\left\|\sum_{j=1}^{n} \beta_{j}(x) b_{j}\right\|=\infty$.

(V) If $B$ satisfies the hypotheses of Theorem 1 and (2) holds for all $x \in B$, then by Theorem $1\left(b_{n}\right)$ is an unconditional basis for $B$, and in particular $B$ is separable. This result, for $B$ separable, has been announced by William J. Davis, David W. Dean, and Ivan Singer [A.M.S. Notices 17 (1970), 437].

(VI) The argument of the second paragraph of Theorem 1, in the context of Harmonic Analysis, is due to Figá-Talamanca (see [4], p. 347).

3. Biorthogonal Decompositions. We wish now to state a similar result concerning biorthogonal decompositions; first some preliminaries:

Given a Banach space $B$ and a collection $\left\{M_{\alpha}, P_{\alpha}\right\}_{\alpha \in A}$ we say that $\left\{M_{\alpha}, P_{\kappa}\right\}$ is a biorthogonal decomposition in $B$ if for each $\alpha \in A, M_{\alpha}$ is a closed linear subspace of $B$ and $P_{\alpha}$ is a bounded linear projection of $B$ onto $M_{\alpha}$ with $P_{\alpha}(x)=0$ whenever $x \in M_{\beta}$ and $\beta \neq \alpha$. We say that $\left\{M_{\alpha}, P_{\alpha}\right\}$ is complete if the linear span of $\left\{M_{\alpha}\right\}$ is dense in $B$ and if $P_{\alpha}(x)=0$ for all $\alpha$ implies $x=0$.

Let now the Banach space $B$ and $\left\{M_{\alpha}\right\}_{\alpha \in A}$, a collection of closed linear subspaces of $B$, be given. For $A_{1} \subseteq A$, let $S\left(A_{1}\right)$ denote the closed linear span of $\left\{M_{\alpha}\right\}_{\alpha \in A_{1}}$. We have:

Proposition. Assume $S(A)=B$. There is a complete biorthogonal decomposition $\left\{M_{\kappa}, P_{\alpha}\right\}_{\alpha \in A}$ of $B$, corresponding to $\left\{M_{\alpha}\right\}_{\alpha \in A}$ if and only if both of the following conditions hold: 
(1) $S\left(A_{1}\right) \cap S\left(A \sim A_{1}\right)=(0)$ for all $A_{1} \subseteq A$.

(2) $S(\{\alpha\})+S(A \sim\{\alpha\})=B$ for all $\alpha \in A$.

Proof. The "only if" part is trivial. Suppose now that (1) and (2) hold. Then fixing $\alpha \in A$, (1) and (2) imply that

$$
B=S(\{\alpha\}) \oplus S(A \sim\{\alpha\}) .
$$

Thus letting $P_{\alpha}$ be the projection onto $S(\{\alpha\})$ with kernel $S(A \sim\{\alpha\})$, $P_{\alpha}$ is bounded by the Closed Graph Theorem, whence $\left\{M_{\alpha}, P_{\alpha}\right\}_{\alpha \in A}$ is a. biorthogonal decomposition of $B$.

Now suppose that $x \in B$ and $P_{\alpha}(x)=0$ for all $\alpha$. There exist finite subsets $A_{n} \subseteq A$ and elements $x_{n} \in S\left(A_{n}\right)$ such that $x_{n} \rightarrow x$. Since $\lim _{n \rightarrow \infty} P_{\alpha}\left(x_{n}\right)=P_{\alpha}(x)=0$ for all $\alpha \in A$, we claim that one can choose a subsequence $\left(n_{k}\right)$, subsets $B_{k} \subseteq A_{n_{k}}$ and elements $y_{k} \in S\left(B_{k}\right)$ such that $B_{k} \cap B_{j}=\varnothing$ for $k$ even and $j$ odd, and such that $y_{k} \rightarrow x$. To see this, assume (as we may) that $A_{n} \subseteq A_{n+1}$ for all $n$. Put $n_{o}=1$; having chosen $n_{k}$, let $m=\# A_{n_{k}}$ and choose $n_{k+1}>n_{k}$ such that $n \geqq n_{k+1}$ and $\alpha \in A_{n_{k}}$ implies $\left\|P_{\alpha}\left(x_{n}\right)\right\|<(m(k+1))^{-1}$. This defines $\left(n_{k}\right)$; now put $B_{k}=A_{n_{k}} \sim A_{n_{k-1}}$ and $y_{k}=x_{n_{k}}-\sum_{\alpha \in A_{n_{k-1}}} P_{\alpha}\left(x_{n_{k}}\right)$ for $k=1,2, \cdots$.

Let $A_{1}=\cup_{k=1}^{\infty} B_{2 k}$. Then $y_{2 k} \rightarrow x, y_{2 k+1} \rightarrow x$, so

$$
x \in S\left(A_{1}\right) \cap S\left(A \sim A_{1}\right)=(0) .
$$

REMARK: If each $M_{\alpha}$ is finite-dimensional and $S(A)=B$ then (2) is automatically satisfied. Thus a sequence $\left\{b_{n}\right\}_{n \in N}$ in $B$ corresponds to a complete biorthogonal sequence $\left\{\left(b_{n}, \beta_{n}\right)\right\}$ in $B$ if and only if $S(N)=B$ and $S\left(N_{1}\right) \cap S\left(N \sim N_{1}\right)=(0)$ for all $N_{1} \subseteq N$.

Theorem 2. Let $B$ be a Banach space and let $\left\{M_{\alpha}\right\}_{\alpha \in A}$ be a collection of closed separable subspaces with dense span such that

(1) $S\left(A_{1}\right) \cap S\left(A \sim A_{1}\right)=(0)$ for all $A_{1} \subseteq A$.

(2) $S(\{\alpha\})+\$(A \sim\{\alpha\})=B$ for all $\alpha \in A$.

Then $\left\{x \mid \exists x_{\alpha} \in M_{\alpha}\right.$ such that $\Sigma x_{\alpha}$ converges unconditionally to $\left.x\right\}=$ $\cap\left\{S\left(A_{1}\right)+S\left(A \sim A_{1}\right) \mid A_{1} \subseteq A\right\}$.

Proof. By the preceding proposition, $\left\{M_{\alpha}, P_{\alpha}\right\}_{\alpha \in A}$ is a complete biorthogonal decomposition of $B$, where $P_{\alpha}$ is the projection onto $S(\{\alpha\})$ with kernel $S(A \sim\{\alpha\})$. Since $S(A)=B$, if $x \in B$ we have that $P_{\alpha}(x)=0$ for all but a countable number of $\alpha^{\prime}$ s, say $\left\{\alpha_{n}\right\}$. If $x \in \cap\left\{S\left(A_{1}\right)+S\left(A \sim A_{1}\right) \mid A_{1} \subseteq A\right\}$, then given $a \in l_{\infty}, a_{n}=0$ or 1 , by letting $A_{1}=\left\{\alpha_{n} \mid \alpha_{n}=1\right\}$ we have that there exists $y \in B$ such that $P_{\alpha_{n}}(y)=a_{n} P_{\alpha_{n}}(x)$ for all $n$ and $P_{\alpha}(y)=0, \alpha \notin\left\{\alpha_{n}\right\}$. All such $y^{\prime}$ s are contained in the separable Banach space $S\left(\left\{\alpha_{n}^{\prime}\right\}\right)$. With this observation, the proof is similar to the proof of Theorem 1 , with $\left(\beta_{n}\right)$ replaced 
by $\left\{P_{\alpha}\right\}$.

We conclude with several remarks:

(1) Assume that $B$ contains no subspace isomorphic to $l_{\infty}$. Then Theorem 2 admits the following generalization: Let $\left\{M_{\alpha}, P_{\alpha}\right\}_{\alpha \in A}$ be a biorthogonal decomposition of $B$ such that $x \in B$ and $P_{\alpha}(x)=0$ for all $\alpha$ implies $x=0$, and let $x \in B$ be such that for every function $a: A \rightarrow\{0,1\}$ with $a^{-1}\{1\}$ countable, there exists $y \in B$ with $P_{\alpha}(y)=$ $a(\alpha) P_{\alpha}(x)$ for all $\alpha \in A$. Then $P_{\alpha}(x)=0$ for all but countably many $\alpha$ 's, and $\Sigma P_{\alpha}(x)$ converges unconditionally to $x$. The proof proceeds as in the proof of Theorem 1; one deduces that for each countable subset $A_{o}$ of $A, \sum_{\alpha \in A_{0}} P_{\alpha}(x)$ converges unconditionally in norm, from which the conclusion easily follows.

(2) For the special case in which $S\left(A_{1}\right)+S\left(A \sim A_{1}\right)=B$ for all $A_{1} \subseteq A$, Theorem 2 was proven in [1].

(3) Theorem 2 applies to the Banach space $L_{p}(G), 1 \leqq p<\infty$, where $G$ is a compact topological group and each $M_{\alpha}$ is the finitedimensional subspace generated by the character of an irreducible unitary representation of $G$. If $G$ is abelian, a direct proof is available, using the existence of approximate identities for $L_{p}$ which are bounded in the $L_{1}$-norm.

\section{REFERENCES}

1. G. F. Bachelis, Homomorphisms of annihilator Banach algebras, Pacific J. Math., 25 (1968), 229-247.

2. M. M. Day, Normed Linear Spaces, Springer-Verlag, Berlin, 1958.

3. J. Dieudonné, Sur la convergence des suites de mesures de Radon, Anais. Acad. Brasil. Ci., 23 (1951), 21-38, 277-282.

4. A. Figá-Talamanca, Apparteneza a $L^{p}$ delle series di Fourier aleatorie su gruppi non commutativi, Rend. Sem. Mat. Univ. Padova, 39 (1967), 330-348.

5. A. Grothendieck, Sur les applications lineaires faiblement compactes d'espaces du type $C(K)$, Canad. J. Math., 5 (1953), 129-173.

6. M. Kadec and A. Pelczynski, Basic sequences, biorihogonal sequences and norming sets in Banach and Frechet spaces, Studia Math., 25 (1965), 297-323 (in Russian).

7. H. P. Rosenthal, On relatively disjoint families of measures, with some applications to Banach space theory, Studia Math., 37 (1971), 13-36, 311-313.

8. G. Seever, Measures on F-Spaces, Trans. Amer. Math. Soc., 133 (1968), 267-280.

Received February 5, 1970, and in revised form July 17, 1970. The research for the second named author was partially supported by NSF Grant GP-12997.

State University of NeW YoRk at Stony Brook and

University of CALIFornia, Berkeley 



\section{PACIFIC JOURNAL OF MATHEMATICS}

\section{EDITORS}

H. SAMELSON

Stanford University

Stanford, California 94305

C. R. Новву

University of Washington

Seattle, Washington 98105
J. DUGundJI

Department of Mathematics

University of Southern California

Los Angeles, California 90007

RICHARD ARENS

University of California

Los Angeles, California 90024

\section{ASSOCIATE EDITORS}
E. F. BECKENBACH
B. H. NeumanN
F. WOLF
K. YOSHIDA

\section{SUPPORTING INSTITUTIONS}
UNIVERSITY OF BRITISH COLUMBIA
CALIFORNIA INSTITUTE OF TECHNOLOGY
UNIVERSITY OF CALIFORNIA
MONTANA STATE UNIVERSITY
UNIVERSITY OF NEVADA
NEW MEXICO STATE UNIVERSITY
OREGON STATE UNIVERSITY
UNIVERSITY OF OREGON
OSAKA UNIVERSITY
UNIVERSITY OF SOUTHERN CALIFORNIA
STANFORD UNIVERSITY
UNIVERSITY OF TOKYO
UNIVERSITY OF UTAH
WASHINGTON STATE UNIVERSITY
UNIVERSITY OF WASHINGTON
AMERICAN MATHEMATICAL SOCIETY CHEVRON RESEARCH CORPORATION TRW SYSTEMS
NAVAL WEAPONS CENTER 


\section{Pacific Journal of Mathematics}

\section{Vol. 37, No. $1 \quad$ January, 1971}

Gregory Frank Bachelis and Haskell Paul Rosenthal, On unconditionally

converging series and biorthogonal systems in a Banach space .........

Richard William Beals, On spectral theory and scattering for elliptic

operators with singular potentials .........................

J. Lennart (John) Berggren, Solvable and supersolvable groups in which every element is conjugate to its inverse ........................ 21

Lindsay Nathan Childs, On covering spaces and Galois extensions ..........

William Jay Davis, David William Dean and Ivan Singer, Multipliers and

unconditional convergence of biorthogonal expansions..............

Leroy John Derr, Triangular matrices with the isoclinal property ............

Paul Erdős, Robert James McEliece and Herbert Taylor, Ramsey bounds for

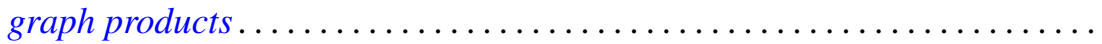

Edward Graham Evans, Jr., On epimorphisms to finitely generated

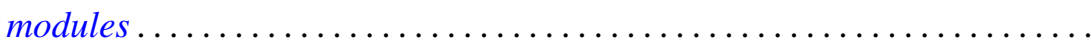

Hector O. Fattorini, The abstract Goursat problem ................. 51

Robert Dutton Fray and David Paul Roselle, Weighted lattice paths .........

Thomas L. Goulding and Augusto H. Ortiz, Structure of semiprime $(p, q)$

radicals ...........................................

E. W. Johnson and J. P. Lediaev, Structure of Noether lattices with join-principal maximal elements ....

David Samuel Kinderlehrer, The regularity of minimal surfaces defined over

slit domains

Alistair H. Lachlan, The transcendental rank of a theory. .

Frank David Lesley, Differentiability of minimal surfaces at the boundary ...

Wolfgang Liebert, Characterization of the endomorphism rings of divisible torsion modules and reduced complete torsion-free modules over complete discrete valuation rings....

Lawrence Carlton Moore, Strictly increasing Riesz norms.

Raymond Moos Redheffer, An inequality for the Hilbert transform ...

James Ted Rogers Jr., Mapping solenoids onto strongly self-entwined,

circle-like continua..........................

Sherman K. Stein, B-sets and planar maps ................... 217

Darrell R. Turnidge, Torsion theories and rings of quotients of Morita

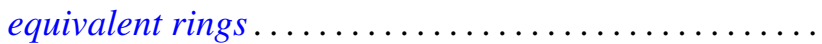

Fred Ustina, The Hausdorff means of double Fourier series and the principle of localization ................................

Stanley Joseph Wertheimer, Quasi-compactness and decompositions for arbitrary relations.

Howard Henry Wicke and John Mays Worrell Jr., On the open continuous images of paracompact $\check{C}$ ech complete spaces... 